\title{
LA ESTRUCTURA DE MODO Y EL SISTEMA DE MODO EN LA CLÁUSULA DEL BRIBRI DE COROMA SEGÚN LA GRAMÁTICA SISTÉMICO-FUNCIONAL
}

\author{
Sara Pacchiarotti
}

\begin{abstract}
RESUMEN
Este artículo analiza la estructura de Modo y el sistema de MODO en la cláusula como intercambio (metafunción interpersonal) según el marco teórico de la gramática sistémico-funcional propuesta por Halliday (2004). En cuanto al sistema paradigmático de MODO (gramaticalización del sistema función de habla), se analiza la realización de las categorías gramaticales (declarativas, interrogativas, imperativas), asociadas a las respectivas categorías socio-semánticas (declaración, preguntas, órdenes), en cuatro textos en lengua bribri (dialecto de Coroma). Finalmente, se analiza la estructura sintagmática de Modo en la cláusula bribri (Modo+Residuo).

Palabras clave: Modo, MODO, bribri, gramática sistémico-funcional.
\end{abstract}

\begin{abstract}
This article seeks to analyze the structure of Mood and the system of MOOD in the clause as interchange (interpersonal metafunction) according to Halliday's systemic functional grammar (2004). With regard to the paradigmatic system of MOOD (grammaticalization of the system of speech acts), the article analyses how grammatical categories (declaratives, interrogatives, imperatives), associated with socio-semantic ones (statements, questions, orders), are construed in four Bribri (Coroma's variation) texts. Finally, the article includes the analysis of syntagmatic Mood structure (Mood + Residue) of the bribri clause.

Key words: Mood, MOOD, bribri, systemic-functional grammar.
\end{abstract}

\section{La lengua bribri y sus hablantes}

El bribri es una lengua perteneciente a la estirpe chibchense (Constenla 1991). El autor indica que dicha estirpe se compone de las siguientes lenguas: paya, rama, guatuso, bribri, cabécar, boruca, tiribí (térraba- teribe), bocotá, movere, dorasque, chánguena, cuna, chimila,

ML. Sara Pacchiarotti. Profesora interina. Escuela de Filología, Lingüística y Literatura. Universidad de Costa Rica.

Correo electrónico: sara_pacchiarotti@yahoo.it

Recepción: 27- 09- 2010

Aceptación: 12- 11- 2010 
cágaba (cogui), guamaca (sancá, marocasero, arsario, malayo, huihua, damana), atanques (cancuama), bíntucua (ica, arhuaco), barí (motilón, dobocubí), tunebo, muisca (chibcha) y duit. De estas, el dorasque, el chánguena, el atanques, el muisca y el duit son lenguas extintas. La estirpe chibchense se extiende desde la costa oriental de Honduras hasta la región central de Colombia. De las lenguas mencionadas, el guatuso, el bribri, el cabécar, el boruca y el térraba se hablan en Costa Rica. El bribri y el cabécar, además, conforman el grupo viceíta.

Los bribris habitan la región sureste de Costa Rica, particularmente ambos de la Cordillera de Talamanca (Jara Murillo 2004a: 90). Del lado del Océano Pacífico, los bribris se ubican en el cantón de Buenos Aires, provincia de Puntarenas, sobre todo en las reservas de Salitre y Cabagra. Del lado del Océano Atlántico, los bribris viven en la Reserva de Talamanca, en el cantón de Talamanca, en la provincia de Limón. Además, se distinguen tres variedades dialectales: Amubre (Talamanca oriental), Coroma (Talamanca occidental) y Salitre (Pacífico Sur). Por lo que atañe al presente artículo, el material de análisis ${ }^{1}$ pertenece exclusivamente al dialecto de Coroma.

\section{La cláusula como intercambio: Modo y MODO según la perspectiva sistémico-funcional}

En el marco de la gramática sistémico-funcional (GSF), uno de los componentes de la cláusula es su organización como evento interactivo, en el que están involucrados un hablante y un público. Según Halliday (1973: 41), la metafunción interpersonal plasma todos los usos del lenguaje destinados a expresar relaciones sociales y personales, incluyendo la totalidad de las formas de intromisión del hablante en la situación y el acto de habla. Así como el componente ideacional proporciona los recursos para la representación de la experiencia, el componente interpersonal proporciona el potencial de significación para todos los usos del lenguaje. La metafunción interpersonal e ideacional, por ende, determinan gran parte del potencial de significación que está incluido en la gramática de todas las lenguas. En la metafunción interpersonal de la cláusula, se pueden distinguir dos roles fundamentales de habla: (i) dar y (ii) pedir. 'Dar' implica 'invitar a alguien a recibir', mientras que 'pedir' implica 'invitar a alguien a dar'. Otra distinción fundamental está relacionada con la naturaleza de la mercancía que está siendo intercambiada: a) bienes y servicios o b) información. En el caso del intercambio de bienes y servicios, el objetivo de un determinado acto de habla es el de obtener que el que escucha haga algo por el que habla. Consecuentemente, la mercancía de intercambio es estrictamente no verbal: lo que se pide es un objeto o bien una acción, usando el lenguaje como vehículo para llevar a cabo el proceso. Sin embargo, en el intercambio de información, el objetivo del que habla es obtener que el oyente diga algo sobre el tema que se está tratando. Lo que se pide es una información: el lenguaje es tanto el medio como el fin y la única respuesta pertinente es una respuesta verbal.

Cuando el lenguaje se utiliza para intercambiar información, la cláusula se transforma en proposición: algo sobre lo que se puede argumentar, algo que puede afirmarse o negarse, aceptarse, contradecirse, etc. Las proposiciones tienen una gramática claramente definida: el lenguaje desarrolla recursos gramaticales para las declaraciones y las preguntas, ya que en ellas el lenguaje es medio y finalidad. Sin embargo, cuando el lenguaje se utiliza para intercambiar bienes y servicios, la cláusula se transforma en propuesta. Los ofrecimientos y las órdenes no pueden ser afirmados o negados, pues no constituyen proposiciones, sino propuestas y, para 
ellas, el lenguaje no desarrolla recursos especiales, dado que las finalidades de las propuestas son típicamente extralingüísticas.

Aunque no exista una correspondencia biunívoca entre ellas, la asociación de categorías gramaticales a categorías socio-semánticas más elevadas es muy natural en el caso de formas gramaticales que se refieren a la expresión de roles sociales y, en particular, a los sistemas que reflejan la estructura social inherente a la situación de habla (Halliday 1973: 56). El sistema principal en este sentido es el sistema de MODO, en el que una cláusula puede ser indicativa o imperativa: al ser indicativa puede ser a su vez declarativa o interrogativa y, al ser interrogativa, puede ser interrogativa de contenido o polar. El MODO sistematiza el conjunto de escogencias mediante el cual el hablante puede asumir uno entre una serie de posibles roles comunicativos, es decir, roles sociales que existen sólo a través del lenguaje, como funciones de la situación de habla. Cabe destacar que en GSF, el MODO es un sistema paradigmático, mientras que Modo es la estructura sintagmática interpersonal de la cláusula, que se compone de Sujeto $\wedge$ Finito.

La estructura del Modo está formada por dos partes: el Sujeto, que es generalmente un grupo nominal, y el operador Finito, que es parte de un grupo verbal. El elemento Finito es un uno de los operadores verbales que puede expresar pasado o modalidad y, dependiendo de la lengua, puede estar fusionado con el verbo léxico. La secuencia Sujeto + Finito sirve para realizar la selección de modo en la cláusula. El resto de la cláusula se define en GSF como Residuo. El Finito es el elemento que circunscribe la deixis interpersonal de una cláusula determinada expresando (i) tiempo primario, es decir pasado, presente o futuro en el momento de la enunciación, y (ii) modalidad. Otra característica concomitante a la finitud es la polaridad, esto es, la escogencia entre positivo y negativo.

En la cláusula como intercambio, por lo tanto, el Sujeto proporciona el resto de lo que se necesita para formar una proposición: algo en relación a lo que la proposición puede ser afirmada o negada. Al tomar en cuenta el intercambio como propuesta, resulta evidente que el Sujeto posee también la responsabilidad del funcionamiento de la cláusula como evento interactivo: el Sujeto típico de un ofrecimiento es el hablante, mientras que el de una orden es la persona a la que el hablante se está dirigiendo. Paralelamente, en una proposición el Sujeto sigue especificando el elemento 'responsable' pero esta vez en términos de la validez ${ }^{2}$ de la información que está siendo enunciada.

El Residuo consiste de tres elementos funcionales: el Predicador, el Complemento y el Adjunto. El Predicador está presente en todas las cláusulas mayores, excepto en las que se elimina por elipsis. Se realiza por medio de un grupo verbal, excluyendo el operador temporal o modal. El Predicador en sí es no finito y existen cláusulas no finitas que contienen un Predicador pero no un Finito. Este elemento tiene una función cuádruple. En primer lugar, especifica la referencia temporal distinta de la que se presenta en el evento de habla: define un tiempo secundario (pasado, presente o futuro) relativo al tiempo primario. En segundo lugar, especifica otros varios aspectos y fases (parecer, tratar, esperar). En tercer lugar, define la voz activa o pasiva y, por último, caracteriza semánticamente el proceso (acción, evento, proceso mental, relación) que se predica acerca del Sujeto.

Un Complemento es un elemento en el Residuo que tiene el potencial de ser Sujeto pero no lo es. En otras palabras, es un elemento al que potencialmente puede atribuirse el estatus interpersonal más elevado de responsabilidad modal, algo que puede constituir el núcleo del argumento. Se realiza típicamente por medio de un grupo nominal. Desde esta perspectiva, 
cualquier grupo nominal que no funcione como Sujeto será un Complemento, incluyendo los grupos nominales encabezados por un adjetivo.

Un Adjunto es un elemento que no posee el potencial para ser elevado al estatus de responsabilidad modal; esto es, no puede convertirse en Sujeto. Esta característica de los Adjuntos implica que no pueden construirse argumentos alrededor de los mismos, ya que, en términos experienciales, los argumentos no pueden construirse alrededor de las Circunstancias, sino alrededor de los Participantes, como el Sujeto y, potencialmente, el Complemento.

A raíz de estas consideraciones, pueden definirse tres grados de elevación interpersonal en la cláusula: el Sujeto, en el que la responsabilidad modal es real; el Complemento, en el que ésta es potencial, y el Adjunto, al que no puede atribuirse ninguna. Un Adjunto es típicamente realizado por un grupo adverbial o una frase preposicional (contiene un Complemento en su estructura interna).

\section{La estructura de Modo y el sistema de MODO en bribri}

\subsection{Cláusulas imperativas}

Halliday y Matthiessen (2006: 485) sugieren que hay que considerar dos distintas nociones para determinar si en las cláusulas imperativas un determinado participante puede funcionar como Sujeto. La primera es la noción experiencial del control del participante en la ejecución de un proceso y la segunda es la noción interpersonal de responsabilidad modal. Ergo, en una cláusula imperativa el Sujeto es el participante responsable de la orden que se está impartiendo: existe un participante al que se le asigna responsabilidad modal, aunque este participante no sea considerado en control del proceso desde un punto de vista experiencial:

\footnotetext{
Thus, the main constraint is an interpersonal one: the modally responsible participant, the Subject, of an imperative clause has to be an interactant - the speaker, the addressee, or the combination of the two: Be quiet! Go to sleep! [...] are all clauses with an implicit addressee Subject, assigned the responsibility of making the command a success, regardless of the participant role. (Halliday y Matthiessen 2006: 486)
}

En este apartado, se analiza el Sujeto desde la perspectiva interpersonal en la cláusula imperativa bribri, partiendo de la estructura Modo + Residuo.

\subsubsection{Sujeto y Complemento: responsabilidad modal y validez dialógica en el intercambio} de información y de bienes y servicios

El Modo de la cláusula imperativa ${ }^{3}$ en bribri varía dependiendo de la transitividad del verbo y de la polaridad. Cuando el verbo es intransitivo y la polaridad es positiva, la estructura del Modo es Sujeto $^{\wedge}$ Predicador $^{\wedge}$ Finito $^{4}$ :

a)

\begin{tabular}{|c|c|c|c|}
\hline $\begin{array}{l}\text { be' } \\
2 S\end{array}$ & \multicolumn{2}{|l|}{$\begin{array}{l}\text { yú } \\
\text { vaya }\end{array}$} & $\begin{array}{l}\text { ie'pa kula' tulèsẽ aula ờmĩk e' ã } \\
\text { 3PL asientos están aula en eso a }\end{array}$ \\
\hline Sujeto & \multicolumn{2}{|c|}{ Predicador ${ }^{\wedge}$ Finito } & Adjunto \\
\hline Modo & Res. & Modo & Residuo \\
\hline \multicolumn{4}{|c|}{ Vaya adonde aquellos, cuyos asientos están en el aula $\left(\mathrm{T} 1^{5}\right)$} \\
\hline
\end{tabular}


Cuando el verbo es transitivo, y la cláusula es afirmativa, el Modo se codifica como Complemento $^{6 \wedge}{ }^{\wedge}$ Predicador ${ }^{\wedge}$ Finito:

b)

\begin{tabular}{|l|l|}
\hline $\begin{array}{l}\text { i yế jkuö } \\
\text { eso papel }\end{array}$ & $\begin{array}{l}\text { táũkã } \\
\text { marque }\end{array}$ \\
\hline Complemento & Predicador ${ }^{\wedge}$ Finito \\
\hline Residuo & Modo \\
\hline \multicolumn{2}{|l|}{ Marque la papeleta $(\mathrm{T} 1)$} \\
\hline
\end{tabular}

El bribri es una lengua con sistema ergativo-absolutivo. En este tipo de sistema, al contrario de las lenguas con sistema nominativo-acusativo, el Sujeto (S) y el Paciente (P) se marcan de la misma forma, mientras que el Agente (A) se marca de manera distinta. Esto se refleja en la estructura Modo + Residuo de las cláusulas imperativas en bribri, independientemente de la polaridad de la cláusula. Las imperativas son la realización gramatical congruente de una orden. El Sujeto, según Halliday, es el elemento que posee la responsabilidad modal en la cláusula, esto es, en el caso de una orden, el elemento responsable de la ejecución de la misma. El concepto de responsabilidad modal define el rol comunicativo adoptado por el hablante:

\footnotetext{
It is present in clauses of all moods, but its significance can perhaps be seen most clearly in the imperative, where the meaning is "request you to..."; here the speaker is requiring some action on the part of the person addressed, but it is the latter who has the power to make this meaning "come true" or otherwise, since he can either obey or disobey. In the usual form of the imperative, this modal entity, or "modal subject" as we may call it, is the listener; and the only option is plus or minus the speaker himself. (Halliday 1970: 190)
}

En la cláusula imperativa bribri, cuando el verbo es intransitivo, el Sujeto es el elemento que posee esta responsabilidad modal: en $B e^{\prime}$ yú (vaya) el destinatario, be', es el que es responsable de ir al lugar que se le indique, y de tal forma, cumplir con la orden impartida por el hablante. Paralelamente, en las imperativas transitivas es el Sujeto elidido, el que tiene la responsabilidad modal en la cláusula. En b), por lo tanto, el Sujeto no es $i$ yë jkuö sino be', aunque esté elidido. Desde la perspectiva ergativo-absolutiva, el Sujeto be' en a) y el Complemento $i$ yë jkü̈ en b) son un mismo participante, el Medio, y se codifican de la misma manera, es decir, sin ningún tipo de marcación morfológica y en posición pre-verbal. El hecho de que en las cláusulas imperativas este participante aparezca en posición pre-verbal refleja que la estructura de la cláusula del bribri es ABS-V como establece Jara Murillo:

\footnotetext{
In sum, the analysis shows that the basic clause structure is: (ERG/OBL) + ABSOLUTIVE + VERB + (OBL/OTHERS), where constituents in parenthesis are structurally optional. This structure serves to encode any proposition composed by a predicate and its related arguments. [...] The basic event is encoded in Bribri discourse as ABSOLUTIVE-VERB, something happened to somebody, and further meaning specifications are given either before or after that basic structure. (1995: 68-70)
}

Lo que determina el estatus de Sujeto (S) en la imperativa intransitiva y el Complemento $(\mathrm{P})$ en la imperativa transitiva es, por ende, su orden en la cláusula, es decir, su ubicación pre-verbal.

En el intercambio de bienes y servicios, es decir en las cláusulas imperativas, el Sujeto es el elemento modalmente responsable. En un intercambio de información, sin embargo, el Sujeto es el elemento sobre el que recae que la validez de la información expresada en una determinada cláusula declarativa. Halliday (2004: 117) especifica que el concepto de validez no debe entenderse en términos de verdadero o falso, sino en relación con el intercambio dialógico, en el que un determinado elemento es establecido, negado, discutido o argumentado. 
El consentimiento o el rechazo de la validez del Sujeto es un rasgo semántico que, según Halliday se negocia en el diálogo. Mientras en las cláusulas imperativas el Sujeto modalmente responsable es el hablante, el destinatario o ambos, en las declarativas un Complemento puede ser elevado al estatus interpersonal de Sujeto, independientemente de que el Complemento sea o no animado:

\begin{abstract}
A Complement is an element within the Residue that has the potential of being a Subject but is not. [...] It is typically realized by a nominal group. So in "the duke gave my aunt that teapot, there are two Complements, my aunt and that teapot. Either of these could function as Subject in the clause: my aunt was given that teapot by the duke and that teapot was given to my aunt by the duke. [...] It will be noted that the Complement covers what are 'objects' as well as what are 'complements' in the traditional school grammar. But that distinction has no place in the interpersonal structure; it is imported from the experiential analysis, that of transitivity. Since the term 'object' is strongly associated with the formal analysis of transitivity, we use Complement as the term for this single element within the Residue (Halliday 2004: 123)
\end{abstract}

Un Complemento desde la perspectiva hallidiana es un Sujeto en potencia, mientras que un Adjunto no puede ser elevado al estatus de Sujeto. El concepto de responsabilidad modal en el sentido de validez de la información en cláusulas declarativas puede entenderse más claramente tomando en cuenta los siguientes ejemplos:

c)

\begin{tabular}{|c|c|c|c|}
\hline $\begin{array}{l}\text { alà tö } \\
\text { niño }\end{array}$ & $\begin{array}{l}\text { ali' } \\
\text { yuca }\end{array}$ & $\begin{array}{c}\text { ñế } \\
\text { comió }\end{array}$ & $\begin{array}{l}\text { ènĩã } \\
\text { hace un rato }\end{array}$ \\
\hline Sujeto & Complemento & Predicador $^{\wedge}$ Finito & Adjunto \\
\hline Modo & Residuo & Modo & Residuo \\
\hline
\end{tabular}

En c), el Sujeto es alà. El Complemento es un Sujeto en potencia en una cláusula declarativa. Ali', al contrario del Adjunto ènĩa a, puede llegar al estatus interpersonal más elevado en la cláusula, como se muestra en la siguiente cláusula:

d)

\begin{tabular}{|c|c|c|}
\hline $\begin{array}{l}\text { ali' } \\
\text { yuca }\end{array}$ & \multicolumn{2}{|c|}{$\begin{array}{l}\text { sẽnè̀wã } \\
\text { se-enfrió }\end{array}$} \\
\hline Sujeto & \multicolumn{2}{|c|}{ Predicador ${ }^{\wedge}$ Finito } \\
\hline Modo & Residuo & Modo \\
\hline \multicolumn{3}{|c|}{ La yuca se enfrió } \\
\hline
\end{tabular}

En d), el Complemento de la cláusula precedente es elevado al estatus de Sujeto. El hablante le asigna al Complemento la función del elemento alrededor del cual se argumenta la cláusula. En otras palabras, ali' es el elemento responsable de sustentar la validez de lo que se está afirmando y, como tal, es el elemento que el hablante considera más a riesgo, es decir, es el candidato más probable del rechazo de la proposición por parte del destinatario. La función del Sujeto en la negociación de la validez entre hablante y destinatario en el caso de intercambio de información se delinea en el siguiente diálogo:

e)

A: Ali' sẽnẽ̀wã

\begin{tabular}{|c|c|c|}
\hline $\begin{array}{l}\text { ali' } \\
\text { yuca }\end{array}$ & \multicolumn{2}{|c|}{$\begin{array}{l}\text { sẽnềwã } \\
\text { se-enfrió }\end{array}$} \\
\hline Sujeto & \multicolumn{2}{|c|}{ Predicador $\wedge$ Finito } \\
\hline Modo & Residuo & Modo \\
\hline
\end{tabular}


f)

B: Kề, ali’ kề sẽnềwã, ali’ bànềwã

\begin{tabular}{|c|c|c|c|c|c|c|}
\hline $\begin{array}{l}\text { kề } \\
\text { NEG }\end{array}$ & $\begin{array}{c}\text { ali' } \\
\text { yuca }\end{array}$ & $\begin{array}{l}\text { kề } \\
\text { NEG }\end{array}$ & $\begin{array}{l}\text { sẽnềwã } \\
\text { se-enfrió }\end{array}$ & $\begin{array}{l}\text { ali' } \\
\text { yuca }\end{array}$ & \multicolumn{2}{|c|}{$\begin{array}{l}\text { bànềwã } \\
\text { está-caliente }\end{array}$} \\
\hline Adjunto & Sujeto & Finito & Predicador ${ }^{\wedge}$ Finito & Sujeto & \multicolumn{2}{|c|}{ Predicador $^{\wedge}$ Finito } \\
\hline \multicolumn{3}{|c|}{ Modo } & Residuo & & Residuo & Modo \\
\hline
\end{tabular}

La función del Sujeto en las cláusulas imperativas y declarativas estriba, en ambos casos, en el concepto de responsabilidad modal:

\begin{abstract}
It is perhaps easier to see the principle of responsibility in a proposal (a goods- $\&$-services clause), where the Subject specifies the one that is actually responsible for realizing the offer or command. [...] The typical Subject of an offer is the speaker, and that of a command is the person being addressed. [...] This role is clearly recognizable in the case of offers and commands; but it is the same principle that is at work in statements and questions. Here too the Subject specifies the 'responsible' element; but in a proposition this means the one on which the validity of the information is made to rest. (Halliday, 2004: 117)
\end{abstract}

En cuanto a la responsabilidad modal en las cláusulas imperativas, siendo estas la realización congruente de una orden, el Agente que tiene la función de iniciar deliberadamente el evento que se le ordena y el Sujeto que tiene la responsabilidad modal de que la orden se cumpla, es el destinatario, es decir, la persona a quien la orden esté dirigida en un determinado tiempo y espacio deíctico. Por ende, es el destinatario, esto es, el Sujeto elidido de la imperativa transitiva, el que tiene la responsabilidad modal en la cláusula, aunque no esté presente en la estructura de Modo.

Sin embargo, también se han encontrado ejemplos en los que el Sujeto está presente en la cláusula imperativa transitiva:

g)

\begin{tabular}{|l|l|l|l|}
\hline $\begin{array}{l}\text { be' } \\
2 \mathrm{~S}\end{array}$ & $\begin{array}{l}\text { tsuru' } \\
\text { cacao }\end{array}$ & \multicolumn{2}{l|}{$\begin{array}{l}\text { jkuố } \\
\text { tueste }\end{array}$} \\
\hline Sujeto & Complemento & \multicolumn{2}{|c|}{ Predicador^Finito } \\
\hline \multicolumn{2}{|l|}{ Modo Residuo } & Modo \\
\hline \multicolumn{2}{|l}{ "Tueste el cacao" (T4) } \\
\hline
\end{tabular}

h)

\begin{tabular}{|c|c|c|}
\hline $\begin{array}{l}\text { be' } r \\
2 S \text { ERG }\end{array}$ & $\begin{array}{l}\text { krò } \\
\text { pollo }\end{array}$ & $\begin{array}{l}\text { yulố } \\
\text { busque }\end{array}$ \\
\hline Sujeto & Complemento & Predicador $^{\wedge}$ Finito \\
\hline Modo & Residuo & Modo \\
\hline
\end{tabular}

En ambos ejemplos, el Sujeto aparece explícito en la cláusula imperativa transitiva: el primero no muestra marcación de ergativo, mientras que el segundo sí. Esto se debe al hecho de que el Sujeto explícito es un elemento opcional de la cláusula (cf. Jara 1995: 68).

Cabe destacar, además, que en ambos casos, no es el hablante el que está enunciando las cláusulas en el texto, pues estas constituyen ejemplos de discurso citado. La deixis de la cláusula es, por ende, distinta del 'aquí' y 'ahora' del hablante, puesto que la narradora está 
remitiendo a las palabras que el maestro ceremonial suele pronunciar cuando alguien está en el proceso de convertirse en cuidador/a de las piedras curativas (sio'tãmĩ). Quizás la presencia del Sujeto en estos ejemplos atípicos pueda justificarse por el hecho de que no es el hablante el que está impartiendo la orden, ni tampoco es el que escucha el responsable modal de llevarla a cabo, puesto que se trata de discurso citado.

\subsubsection{Los Adjuntos: distintos grados de involucramiento}

Los Adjuntos circunstanciales son elementos que no pueden ser elevados al estatus de Sujeto:

i)

\begin{tabular}{|c|c|c|c|c|}
\hline o & $\begin{array}{l}\mathrm{i} \\
\text { eso }\end{array}$ & \multicolumn{2}{|c|}{$\begin{array}{l}\text { táũkã } \\
\text { marque-sobre }\end{array}$} & $\begin{array}{l}\text { ulàtska wa } \\
\text { dedo con }\end{array}$ \\
\hline & Complemento & \multicolumn{2}{|c|}{ Predicador ${ }^{\wedge}$ Finito } & Adjunto \\
\hline & \multicolumn{2}{|c|}{ Residuo } & Modo & Residuo \\
\hline & rque con el dec & & & \\
\hline
\end{tabular}

En i), ulàtska wa constituye un Adjunto circunstancial. Esto implica que no pueden construirse argumentos alrededor de los Adjuntos o Circunstancias, en términos experienciales, mas sí alrededor de los Participantes, como el Sujeto o, potencialmente, el Complemento:

j)

\begin{tabular}{|c|c|c|}
\hline $\begin{array}{l}\text { ie'pa ã } \\
\text { 3PL a }\end{array}$ & $\begin{array}{l}\text { be' yế jkuö } \\
2 S \quad \text { papel }\end{array}$ & $\begin{array}{l}\text { kàchố } \\
\text { enseñe }\end{array}$ \\
\hline Adjunto & Complemento & Predicador ${ }^{\wedge}$ Finito \\
\hline \multicolumn{3}{|c|}{ Residuo } \\
\hline
\end{tabular}

Ie'pa ã en j) se trata como un Adjunto en GSF, al igual que ulàtska wa en i). Debido a que la estructura posposicional en que se codifica un Beneficiario como ie'pa a se considera un Adjunto en términos interpersonales, la presencia de una posposición (ã) no permite que sea elevado al estatus de Sujeto. Sin embargo, Halliday y Matthiessen (2006: 167) introducen el término participación para indicar que el estatus de un determinado participante en la gramática de la cláusula no es absoluto, sino gradual. El grado de participación depende a su vez del grado de involucramiento: una entidad puede interpretarse en una figura, en términos experienciales, como un participante o como una circunstancia. El hecho de que se interprete como un participante indica un mayor grado de involucramiento $\mathrm{y}$, consecuentemente, de participación. Las diferencias en los grados de involucramiento tienen repercusiones en la metafunción interpersonal:

\footnotetext{
The difference in the degree of involvement is also reflected in the extent to which an element is available for a critical role in the interpersonal metafunction. Prototypically, as we have noted, participants can be assigned the status of Subject, being made to carry the burden of an argument, whereas circumstances cannot. (Halliday y Matthiessen 2006: 173)
}

Desde esta perspectiva, los participantes y las circunstancias constituyen un continuum que no tiene límites definidos, aunque la gramática establezca una división entre ellos: un participante es típicamente un grupo nominal, mientras que una circunstancia es, en el caso del bribri, una frase posposicional. Sin embargo, los autores argumentan que, debido al 
continuum que existe entre participantes y circunstancias, se pueden distinguir tres tipos de categorías mixtas: (i) participantes que parecen circunstancias; (ii) circunstancias que parecen participantes y (iii) pares en los que un elemento es una circunstancia, el otro es un participante y la diferencia entre los dos es mínima.

Retomando los dos ejemplos precedentes, i tã $\tilde{u} k a$ ulàtska wa (marque con el dedo) y ie’pa ã be' yё' jkuö kàchö' (a ellos enséñeles su cédula), aunque ambos se consideren Adjuntos desde la perspectiva interpersonal y ambos sean frases posposicionales, ie'pa ã implica un grado de participación e involucramiento mayor que ulàtska wa, pues representa un caso oblicuo, es decir, un participante que parece una circunstancia por preceder una posposición, mientras que ulàtska wa constituye una circunstancia de modo (cf. Halliday 2004: 262).

\subsubsection{La polaridad negativa en las cláusulas imperativas}

En cuanto a la polaridad en las cláusulas imperativas, Constenla Umaña et al. (1998: 77) indican que en las imperativas negativas, la forma imperativa del verbo se reemplaza por el infinitivo y la presencia del ergativo es obligatoria. Ergo, la polaridad incide en la estructura Modo + Residuo al eliminar el elemento Finito cuando la cláusula es imperativa y negativa. Sin embargo, dos ejemplos de imperativas negativas en el texto Mal agüero del gallo muestran un patrón distinto que, por lo menos en el dialecto de Coroma, indica que posiblemente haya otras formas para la realización de una cláusula imperativa negativa. En estos ejemplos el verbo no aparece en infinitivo y no se da la presencia del ergativo:

k)

\begin{tabular}{|l|l|l|l|}
\hline $\begin{array}{l}\text { kề } \\
\text { NEG }\end{array}$ & \multicolumn{1}{|c|}{$\begin{array}{l}\text { klàtska } \\
\text { uñas }\end{array}$} & \multicolumn{2}{|c|}{$\begin{array}{l}\text { táa } \\
\text { corte }\end{array}$} \\
\hline Finito & Complemento & Predicador $\wedge$ Finito \\
\hline Modo & Residuo & Modo \\
\hline No le corte las uñas (T3) & \\
\hline
\end{tabular}

1)

\begin{tabular}{|l|l|l|l|}
\hline $\begin{array}{l}\text { kề } \\
\text { NEG }\end{array}$ & \multicolumn{1}{|c|}{$\begin{array}{l}\text { ñố } \\
\text { tripas }\end{array}$} & \multicolumn{1}{|c|}{$\begin{array}{l}\text { biátsã } \\
\text { saque }\end{array}$} \\
\hline Finito & Complemento & Predicador ${ }^{\wedge}$ Finito \\
\hline Modo & Residuo & & Modo \\
\hline \multicolumn{2}{|l}{} \\
\hline
\end{tabular}

En estos ejemplos de imperativas negativas, la polaridad, expresada por $k \dddot{e}^{`}$ es parte del Finito, elemento que puede expresar deixis temporal, modal o bien polaridad. Sin embargo, la polaridad negativa parece no influir en el Predicador, que no aparece en infinitivo.

\subsubsection{Deicticidad en las cláusulas imperativas}

Por lo que se refiere a la deicticidad expresada por el Finito fusionado con el Predicador en los verbos en bribri, cabe destacar que este elemento no tiene función deíctica modal o temporal para el MODO imperativo, aunque esté codificado gramaticalmente en la lengua por medio de la caída de la terminación de infinitivo (-k) y tono descendente en la vocal temática del verbo. En las cláusulas imperativas, tanto afirmativas como negativas, lo que crea la deixis del evento como interacción es el Sistema de SUJETO/PERSONA. 
3.2. La estructura de Modo en las cláusulas declarativas e interrogativas: realizaciones del Sistema de MODO e influencia del Sistema de TRANSITIVIDAD

\subsubsection{Cláusulas declarativas}

La estructura del Modo de las cláusulas declarativas transitivas en bribri tiene distintas realizaciones:

(i) Sujeto $^{\wedge}$ Complemento $^{\wedge}$ Predicador $^{\wedge}$ Finito:

$\mathrm{m})$

\begin{tabular}{|l|l|l|l|}
\hline $\begin{array}{l}\text { be' tö } \\
\text { 2S ERG }\end{array}$ & $\begin{array}{l}\text { i } \\
\text { eso }\end{array}$ & $\begin{array}{l}\text { chèke } \\
\text { dice }\end{array}$ \\
\hline Sujeto & Complemento & Predicador ${ }^{\wedge}$ Finito \\
\hline Modo & Residuo & Modo & Residuo \\
\hline \multicolumn{4}{|l}{} \\
\hline
\end{tabular}

(ii) Complemento $^{\wedge}$ Predicador $^{\wedge}$ Finito $^{\wedge}$ Sujeto:

n)

\begin{tabular}{|l|l|l|}
\hline $\begin{array}{l}\text { piña balé } \\
\text { piña chicha }\end{array}$ & $\begin{array}{l}\text { yawè } \\
\text { hace }\end{array}$ & $\begin{array}{l}\text { i di } \\
\text { 3S ERG }\end{array}$ \\
\hline Complemento & Predicador ${ }^{\wedge}$ Finito & Sujeto \\
\hline Residuo & Residuo & Modo \\
\hline \multicolumn{2}{|l}{ El puede hacer chicha de piña $(\mathrm{T} 2)$} \\
\hline
\end{tabular}

Desde el punto de vista experiencial, m) es una cláusula verbal, mientras que n) es una cláusula material. Desde el punto de vista interpersonal, los participantes marcados de estas cláusulas (be tö, $i$ di) representan los Sujetos en la cláusula como intercambio, es decir el elemento al que se le atribuye el estatus de responsabilidad modal más elevado. Siendo cláusulas declarativas, el concepto de responsabilidad modal del Sujeto debe entenderse según Halliday (2004: 117) de la siguiente manera: el Sujeto es el elemento en referencia al cual la proposición puede ser afirmada o negada. En el ejemplo m), be' es la entidad a la que se atribuye la validez de lo que se está afirmando y, por lo tanto, también la que está más a riesgo, en el evento interactivo, de ser la razón por la que el oyente rechace la validez de la proposición.

$\mathrm{N}$ ) representa un ejemplo del hecho de que el orden básico de los constituyentes en bribri es ABS-V. La posición en la que aparezca el Sujeto puede variar, mientras que la posición del Complemento (absolutivo) es inamovible. Cabe observar que, independientemente de la transitividad del verbo, la estructura Modo + Residuo es, en la mayoría de los casos, discontinua, pues el Predicador está fusionado con el Finito y el Complemento (absolutivo) en bribri aparece obligatoriamente antes del verbo e interrumpe la secuencia de Sujeto + Finito perteneciente al Modo.

En las cláusulas declarativas intransitivas, la estructura del Modo es Sujeto $^{\wedge}$ Predicador^Finito:

o)

\begin{tabular}{|c|c|c|c|}
\hline $\begin{array}{l}\mathrm{ie}^{\prime} \\
3 \mathrm{~S}\end{array}$ & \multicolumn{2}{|c|}{$\begin{array}{l}\text { tcháshkal } \\
\text { escapó }\end{array}$} & $\begin{array}{l}\text { bitũ } \\
\text { hacia-acá }\end{array}$ \\
\hline Sujeto & \multicolumn{2}{|c|}{ Predicador ${ }^{\wedge}$ Finito } & Adjunto \\
\hline Modo & Residuo & Modo & Residuo \\
\hline
\end{tabular}


En el ejemplo o), el Sujeto $i e^{\prime}$ no está marcado, pues es el único elemento al que se le puede atribuir el valor de responsabilidad modal puesto que la cláusula es intransitiva. Esto implica que en bribri la responsabilidad modal se hace explícita sólo cuando hay más de un participante, es decir, cuando la cláusula es transitiva.

En la construcción tso' más infinitivo ${ }^{8}$, el Finito no está fusionado con el Predicador y la estructura Modo + Residuo es continua:

p)

\begin{tabular}{|l|l|l|l|}
\hline e' tã $^{\prime}$ & $\begin{array}{l}\text { sa' } \\
1 \mathrm{PL}\end{array}$ & $\begin{array}{l}\text { tso' } \\
\text { estamos }\end{array}$ & $\begin{array}{l}\text { kốpàkök } \\
\text { conversar }\end{array}$ \\
\hline & Sujeto & Finito & Predicador \\
\hline & \multicolumn{2}{|c|}{ Modo } & Residuo \\
\hline \multicolumn{2}{|c|}{ Entonces nos pusimos a conversar (T2) }
\end{tabular}

En este caso, la estructura de Modo + Residuo es Sujeto^Finito^Predicador. Esta estructura también se presenta con otros auxiliares de posición como têr. Cuando la cláusula es transitiva, el Finito precede al Complemento:

q)

\begin{tabular}{|l|l|l|l|l|}
\hline e' tã & $\begin{array}{l}\text { i } \\
\text { 3PL }\end{array}$ & $\begin{array}{l}\text { tếrkerak } \\
\text { solían }\end{array}$ & $\begin{array}{l}\text { nãmầ } \\
\text { pez }\end{array}$ & $\begin{array}{l}\text { kalíũk } \\
\text { enganchar }\end{array}$ \\
\hline & Sujeto & Finito & Complemento & Predicador \\
\hline & Modo & Residuo \\
\hline \multicolumn{2}{l}{ Solían ir a pescar (T2) }
\end{tabular}

La polaridad en las cláusulas que presentan la construcción tso’ más infinitivo afecta morfológicamente el Finito, que cambia de tso' a kũ , al igual que en las cláusulas en las que tso' tiene valor existencial:

r)

\begin{tabular}{|c|c|c|c|c|c|}
\hline $\begin{array}{l}b^{\prime} \\
2 S\end{array}$ & $\begin{array}{l}\text { lè } \\
\text { tal vez }\end{array}$ & $\begin{array}{l}\text { ye' } \\
1 S\end{array}$ & $\begin{array}{l}\text { lè } \\
\text { tal vez }\end{array}$ & $\begin{array}{l}\text { kề kũ } \\
\text { NEG estemos }\end{array}$ & $\begin{array}{l}\text { iã } \\
\text { más }\end{array}$ \\
\hline Sujeto & $\begin{array}{l}\text { Adjunto } \\
\text { modo }\end{array}$ & Sujeto & $\begin{array}{l}\text { Adjunto } \\
\text { modo }\end{array}$ & Finito ^ Predicador & $\begin{array}{l}\text { Adjunto } \\
\text { Modo }\end{array}$ \\
\hline \multicolumn{5}{|r|}{ Residuo } & Modo \\
\hline
\end{tabular}

\subsubsection{Cláusulas interrogativas}

En las interrogativas de contenido, la estructura del Modo es Elemento Interrogativo^ ${ }^{\wedge}$ Sujeto $^{\wedge}$ Complemento $^{\wedge}$ Predicador $^{\wedge}$ Finito:

s)

\begin{tabular}{|l|l|l|l|l|}
\hline $\begin{array}{l}\text { wềs } \\
\text { cómo }\end{array}$ & $\begin{array}{l}\text { síkuapa tö } \\
\text { gente-blanca ERG }\end{array}$ & $\begin{array}{l}\text { balo' } \\
\text { chicha }\end{array}$ & $\begin{array}{l}\text { yawè? } \\
\text { hace }\end{array}$ \\
\hline $\begin{array}{l}\text { Elemento } \\
\text { interrogativo }\end{array}$ & Sujeto & Complemento & \multicolumn{2}{|l|}{ Predicador ^Finito } \\
\hline Residuo & Modo & \multicolumn{2}{|c|}{ Residuo } & Modo \\
\hline ¿Cómo los blancos hacen chicha? (T2) \\
\hline
\end{tabular}


Al igual que en las declarativas, la posición del Sujeto puede variar cuando la cláusula es transitiva, como en el ejemplo t).

En este caso, el elemento interrogativo coincide con el Complemento (absolutivo) y la estructura Modo + Residuo es Complemento/Elemento Interrogativo ${ }^{\wedge}$ Predicador $^{\wedge}$ Finito $^{\wedge}$ Sujeto:

t)

\begin{tabular}{|l|l|l|}
\hline $\begin{array}{l}\text { i } \\
\text { qué }\end{array}$ & $\begin{array}{l}\text { yawè } \\
\text { hace }\end{array}$ & $\begin{array}{l}\text { be' rö? } \\
\text { 2S ERG }\end{array}$ \\
\hline $\begin{array}{l}\text { Complemento/ } \\
\text { Elemento interrogativo }\end{array}$ & Predicador $\wedge$ Finito & Sujeto \\
\hline Residuo & Modo \\
\hline ¿Qué está haciendo? (T2) & \multicolumn{2}{|l}{} \\
\hline
\end{tabular}

En las intransitivas, se presenta la misma estructura que en las declarativas y lo único que las distingue es la presencia del elemento interrogativo:

u)

\begin{tabular}{|l|l|l|l|}
\hline $\begin{array}{l}\text { wế } \\
\text { dónde }\end{array}$ & $\begin{array}{l}\text { ye' } \\
\text { 1S }\end{array}$ & $\begin{array}{l}\text { tso'? } \\
\text { estoy }\end{array}$ \\
\hline $\begin{array}{l}\text { Elemento } \\
\text { interrogativo }\end{array}$ & Sujeto & Predicador ^Finito \\
\hline Residuo & Modo & Residuo & Modo \\
\hline ¿Adónde estoy? (T2) & \multicolumn{2}{|l}{} \\
\hline
\end{tabular}

Las interrogativas polares (sí/no) mantienen la misma estructura de Modo de una declarativa. Lo que las distingue de estas últimas es la entonación:

V)

\begin{tabular}{|l|l|l|l|l|l|}
\hline ahora & $\begin{array}{l}\text { ie' } \\
3 S\end{array}$ & $\begin{array}{l}\text { kề } \\
\text { NEG }\end{array}$ & $\begin{array}{l}\text { làla } \\
\text { niños }\end{array}$ & $\begin{array}{l}\text { ò̀tẽ? } \\
\text { dejó }\end{array}$ \\
\hline & Sujeto & Finito & Complemento & \multicolumn{2}{|l|}{ Predicador ^Finito } \\
\hline & Modo & \multicolumn{2}{|c|}{ Residuo } & Modo \\
\hline
\end{tabular}

\subsubsection{Cláusulas exclamativas}

Las cláusulas exclamativas presentes en los textos no presentan una estructura de Modo distintiva. En la totalidad de las cláusulas encontradas en los textos, las exclamativas se realizan por medio de cláusulas declarativas:

W)

\begin{tabular}{|l|l|l|}
\hline $\begin{array}{l}\text { e' } \\
\text { ese }\end{array}$ & $\begin{array}{l}\text { katèwã } \\
\text { podríamos-comer }\end{array}$ & $\begin{array}{l}\text { sa' rö! } \\
\text { 1PL ERG }\end{array}$ \\
\hline Complemento & Predicador `Finito & Sujeto \\
\hline Residuo & Modo \\
\hline \multicolumn{2}{|l}{ iNos lo podríamos comer! (T2) } \\
\hline
\end{tabular}

Según el informante Alí García Segura (comunicación personal), la exclamación se expresa en bribri en las narraciones, para enfatizar la ocurrencia de un determinado evento, la realización de una acción o quien la realiza. Sin embargo, el Sistema del MODO en bribri no cuenta con recursos gramaticales distintivos para la exclamatividad. 


\subsection{MODO y Modo}

La siguiente Tabla indica las posibles realizaciones sintagmáticas de la estructura Modo + Residuo, según la escogencia paradigmática del MODO:

Tabla 3.1. MODO (paradigmático) y Modo (sintagmático) en cláusulas transitivas

\begin{tabular}{|c|c|c|c|c|}
\hline Modo & & & MODO & \\
\hline & claúsulas & DECLARATIVO & IMPERATIVO & INTERROGATIVO \\
\hline $\begin{array}{c}\mathbf{M} \\
\mathbf{0}\end{array}$ & & $\begin{array}{l}\text { Sujeto^}^{\wedge} \\
\text { Complemento^ }^{\wedge} \\
\text { Predicador^}^{\wedge} \text { Finito } \\
\rightarrow \\
\text { be' tö i chèke } \\
2 \text { S ERG eso dice } \\
\text { Usted dice eso } \\
(\mathrm{T} 2)\end{array}$ & $\begin{array}{l}\text { Complemento }^{\wedge} \\
\text { Predicador^ } \\
\text { Finito } \\
\rightarrow \\
\text { i yế jkuö tấukã } \\
\text { eso papel marque } \\
\text { Marque la papeleta } \\
(\mathrm{T} 1)\end{array}$ & $\begin{array}{l}\text { Elemento } \\
\text { Interrogativo^}^{\wedge} \\
\text { Sujeto^ }^{\wedge} \\
\text { Complemento^}^{\wedge} \\
\text { Predicador^Finito } \\
\rightarrow \\
\text { wềs síkuapa tö } \\
\text { como gente blanca ERG } \\
\text { balo' yawè? } \\
\text { chicha hace } \\
\text { ¿Cómo los blancos } \\
\text { hacen chicha? } \\
\text { (T2) }\end{array}$ \\
\hline $\begin{array}{l}\text { d } \\
\text { o }\end{array}$ & & $\begin{array}{l}\text { Complemento }^{\wedge} \\
\text { Predicador^}^{\wedge} \text { Finito }^{\wedge} \\
\text { Sujeto } \\
\rightarrow \\
\text { piña balé yawè i di. } \\
\text { piña chicha hace } 3 \text { S ERG } \\
\text { El puede hacer chicha } \\
\text { de piña } \\
\text { (T2) }\end{array}$ & & $\begin{array}{l}\text { Complemento/ } \\
\text { Elemento } \\
\text { Interrogativo^} \\
\text { Predicador^Finito^ } \\
\text { Sujeto } \\
\rightarrow \quad \\
\text { i yawè be' rö? } \\
\text { qué hace } 2 \mathrm{~S} \text { ERG } \\
\text { ¿Qué está haciendo? } \\
\text { (T2) }\end{array}$ \\
\hline & & $\begin{array}{l}\text { Sujeto`Finito^}^{\wedge} \\
\text { Complemento^ }^{\wedge} \\
\text { Predicador }^{-} \\
\rightarrow \\
\text { i tếrkerak nãmã̀ kalíũk. } \\
\text { 3PL solían pez } \\
\text { enganchar }\end{array}$ & & $\begin{array}{l}\text { Sujeto / Elemento } \\
\text { Interrogativo }^{\wedge} \\
\text { Complemento }^{\wedge} \\
\text { Predicador }{ }^{\wedge} \text { Finito } \\
\text { yì be' dör? } \\
\text { quién } 2 \text { S es } \\
\text { ¿Quién es usted? }\end{array}$ \\
\hline & & $\begin{array}{l}\text { Ellos solían ir a pescar } \\
(\mathrm{T} 2)\end{array}$ & & $\begin{array}{l}\text { Complemento^} \\
\text { Predicador^Finito^}^{\wedge} \\
\text { Sujeto/Elemento } \\
\text { Interrogativo } \\
\rightarrow \\
\text { be' dör yì? } \\
2 \text { S es quién } \\
\text { ¿Quién es usted? }\end{array}$ \\
\hline
\end{tabular}


Tabla 3.2. MODO (paradigmático) y Modo (sintagmático) en cláusulas intransitivas

\begin{tabular}{|c|c|c|c|c|}
\hline \multicolumn{2}{|c|}{ Modo transitividad } & \multicolumn{3}{|c|}{ MODO } \\
\hline \multirow{3}{*}{$\begin{array}{c}\text { M } \\
\mathbf{o} \\
\mathbf{d} \\
\mathbf{o}\end{array}$} & \multirow{3}{*}{$\begin{array}{l}\text { claúsulas } \\
\text { intransitivas }\end{array}$} & DECLARATIVO & IMPERATIVO & INTERROGATIVO \\
\hline & & $\begin{array}{l}\text { Sujeto^ }^{\wedge} \text { Predicador } \\
\text { Finito^}^{\wedge} \\
\rightarrow \\
\text { ye' kapákwã } \\
\text { 1S quiero-dormir } \\
\text { Tengo sueño } \\
\text { (T2) }\end{array}$ & \multirow[t]{2}{*}{$\begin{array}{l}\text { Sujeto` }^{\wedge} \text { Predicador }^{\wedge} \\
\text { Finito } \\
\rightarrow \\
\text { be' yú } \\
2 \text { S vaya } \\
\text { Vaya } \\
\text { (T1) }\end{array}$} & \multirow[t]{2}{*}{$\begin{array}{l}\text { Elemento } \\
\text { Interrogativo^ }^{\wedge} \\
\text { Sujeto^}^{\wedge} \\
\text { Predicador }^{\wedge} \text { Finito } \\
\rightarrow \\
\text { wế ye' tso'? } \\
\text { donde } 1 \mathrm{~S} \text { estoy } \\
\text { ¿Dónde estoy? } \\
\text { (T2) }\end{array}$} \\
\hline & & $\begin{array}{l}\text { Sujeto }^{\wedge} \\
\text { Finito }^{\wedge} \text { Predicador } \\
\rightarrow \quad \text { sa' tso' kốpàkök } \\
1 \mathrm{PL} \text { estamos conversar } \\
\text { Nos pusimos a } \\
\text { conversar } \\
(\mathrm{T} 2)\end{array}$ & & \\
\hline
\end{tabular}

\section{Conclusiones}

La estructura sintagmática del Modo en bribri es fundamentalmente discontinua, puesto que los elementos que constituyen el Modo (Sujeto^ ${ }^{\wedge}$ Finito) y el Residuo (Complemento^ Predicador^ ${ }^{\wedge}$ Adjunto) se distribuyen de manera heterogénea dentro de la cláusula. Esto se debe principalmente al hecho de que el bribri es una lengua de orden rígido ABS-V (Jara 1995: 68). El absolutivo, cuando es objeto directo, se denomina Complemento en la teoría sistémicofuncional y se clasifica como un elemento perteneciente a la estructura del Residuo. En bribri, sin embargo, la posición del Complemento es obligatoriamente pre-verbal y esto determina la discontinuidad entre el Modo y el Residuo. Cabe destacar que la perspectiva sistémico-funcional que se ha tomado en cuenta para este análisis se aplica al inglés (Halliday, 2004), una lengua SVO y que, debido a este tipo de orden, el Complemento se relega a la estructura de Residuo de la cláusula. Siendo el bribri una lengua OV, la posición pre-verbal del Complemento es la razón fundamental por la cual la estructura modal de la cláusula en esta lengua suele ser discontinua.

Otro punto central en la discontinuidad de la estructura sintagmática es el Finito que, excepto en las construcciones en las que aparece separado del Predicador (i.e: $t$ so' + infinitivo), aparece en la mayoría de los casos fusionado con este. Por último, el hecho de que el bribri sea una lengua de orden rígido ABS-V implica que la posición del Sujeto es relativamente libre, con lo cual se dan casos en los que aparece antes del Complemento y otros en los que aparece después del Predicador ${ }^{\wedge}$ Finito.

La estructura sintagmática Modo+Residuo en bribri presenta dos elementos cuyo orden en la cláusula es fijo: Complemento^Predicador. La posición del Sujeto es flexible en bribri. Jara (2002) afirma que el bribri es una lengua OV con sistema de marcación de casos absolutivoergativo: el ergativo puede aparecer antes o después de la secuencia absolutivo - verbo (ergabs-V o abs-V-erg). En cuanto a la posición del Sujeto ergativo, la autora argumenta lo siguiente: 
La posición de la FN ergativa, sea delante o después de abs-V, parece estar determinada por la organización del discurso. Quiero sugerir que cuando el participante codificado como ergativo es más prominente/importante/relevante en el discurso en comparación con el participante codificado como absolutivo, aquel tiende a ser preverbal; en caso contrario tiende a ser posverbal. (Jara 2002: 118)

El Sistema de TRANSITIVIDAD no influye en el Sistema de MODO, sino en las realizaciones sintagmáticas de la estructura Modo. Lo que diferencia una cláusula declarativa transitiva de una imperativa transitiva es la flexión verbal de modo indicativo e imperativo respectivamente. En ambas, el Sujeto puede estar elidido. Una cláusula declarativa intransitiva tiene la misma estructura Modo + Residuo que una cláusula imperativa intransitiva, excepto en los casos que presentan construcciones con tso' u otros auxiliares de posición + infinito complementario, que pueden aparecer sólo en cláusulas declarativas, por lo que no tienen modo imperativo, en las que el Finito precede al Predicador.

En cuanto a las cláusulas interrogativas, lo que las distingue de otras escogencias del MODO es la presencia de un elemento interrogativo, que puede estar fusionado con el Complemento o el Sujeto. Al igual que en las declarativas transitivas, en las interrogativas transitivas, la posición del Sujeto puede variar. Al igual que en las exclamativas, como se ha dicho, las interrogativas polares no tienen una estructura distintiva en bribri, rasgo muy común a muchas lenguas, sino que suelen realizarse por medio de cláusulas declarativas con una entonación particular. Según Constenla Umaña et al. (1998: 18), lo que las diferencia de las declarativas es un ascenso especial de la entonación en determinada sílaba. En cuanto a la posición del elemento interrogativo en las interrogativas de contenido, Constenla Umaña et al. (1998: 19) sostienen que este puede colocarse tanto en la misma posición que le correspondería al elemento que desempeñe la misma función en las cláusulas declarativas, como al inicio de la cláusula. La colocación al inicio es la más frecuente.

A partir de estas consideraciones, el Sistema de TIPO DE MODO en bribri se puede representar como sigue:

Figura 4.1. Sistema de MODO

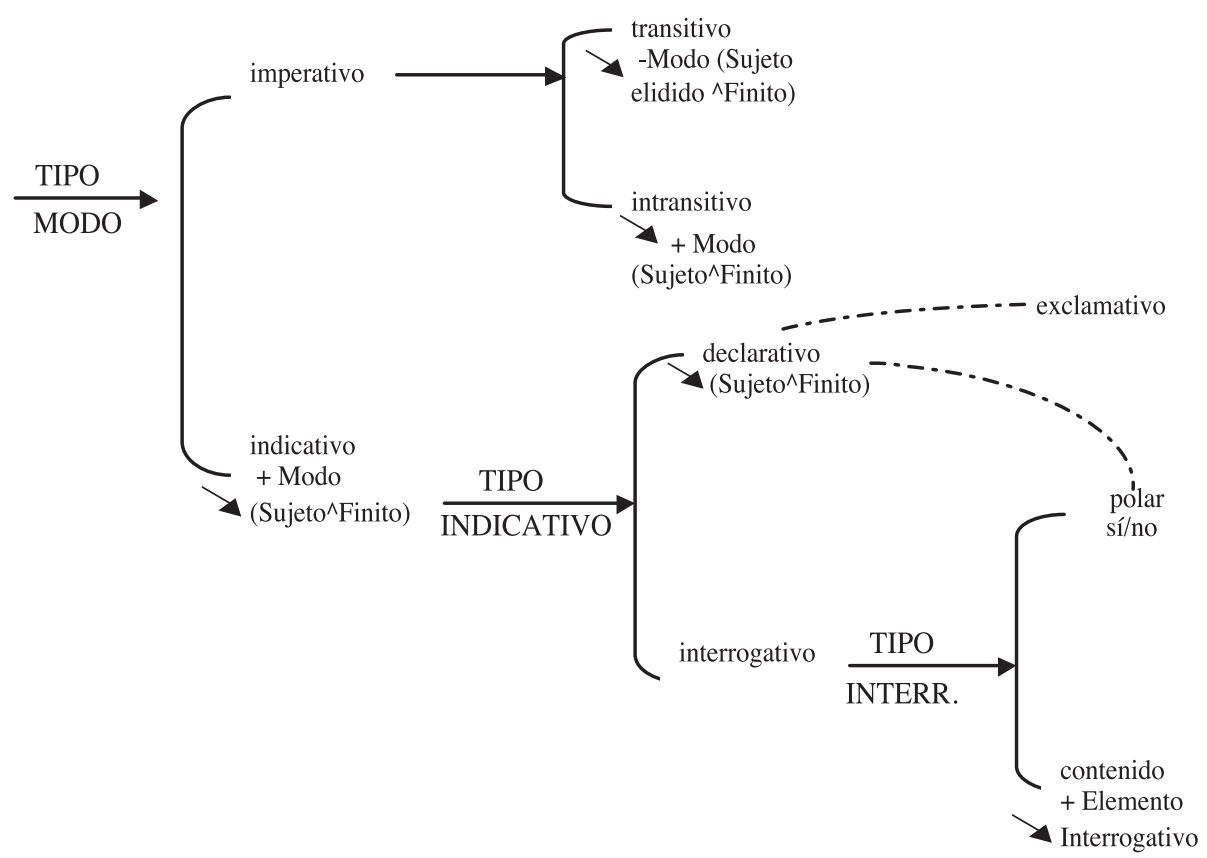




\section{Lista de Abreviaturas}

1S primera persona singular

2S segunda persona singular

3S tercera persona singular

1PL primera persona plural

3PL tercera persona plural

ERG marcador de ergativo

NEG negación

\section{Notas}

1. Los textos que se analizarán en el presente artículo son: "El mal agüero del gallo", un texto narrado por el awá Francisco García que forma parte de I ttè. Historias bribris (Jara Murillo: 1993); "Sĩõ'tãmĩ”, un fragmento de entrevista extraída de Cargos tradicionales del pueblo bribri: Sĩõ'tãmĩ - Óköm - Awá (Jara Murillo y García Segura: 2008); “Cómo se vota” (Wẽ̀s yë’jkuö tawề ke?), un texto instructivo traducido por Alí García Segura; y una conversación informal entre Alí García Segura y su hermano Bahil García Segura, transcrita y analizada por Jara Murillo (1998 y 2004b). Para la división de los textos en cláusulas véase Pacchiarotti (2009).

2. Halliday (2004: 117) especifica que 'validez' no debe entenderse en términos de verdadero o falso, puesto que la semántica no se relaciona en ningún modo con la 'verdad' del enunciado, sino con el consenso acerca de su validez, que es negociado en el diálogo. La noción de la validez se refiere a la argumentabilidad del caso, si se trata de una proposición, o bien a la puesta en efecto si se trata de una propuesta.

3. Cf. Constenla Umaña et al. (1998: 76).

4. Este análisis no contempla la división de las palabras en morfemas, con lo cual, no se ha dividido formalmente el Predicador del Finito en este análisis: "A clause can be analysed into its constituent parts, which are groups and phrases; and these groups and phrases can be analysed into their constituents parts, which are words. (It would also be possible to analyse words into their constituent parts - morphemes, but functional grammar does not take this step)" (Martin, Matthiessen y Painter 1997: 9).

5. El número entre paréntesis se refiere al texto del que fue extraída la cláusula. La numeración de los cuatro textos será la siguiente:

T1: Cómo se vota.

T2: Conversación informal.

T3: El mal agüero del gallo.

T4: Fragmento de la Entrevista a la Siõ’tãmĩ Anastasia Segura.

6. Cabe especificar que el término 'Complemento' no debe entenderse en el sentido tradicional, sino como el elemento en la cláusula que podría ser elevado al estatus de responsabilidad modal del Sujeto, pero que, por alguna razón, no lo es. Siendo el bribri una lengua de sistema ergativo-absolutivo, el término Complemento en este análisis representará siempre el absolutivo, que es un elemento obligatorio en la cláusula bribri.

7. Los ejemplos c), d), e) y f) fueron obtenidos por medio de elicitación con el consultor Alí García Segura.

8. Cf. infinito complementario en Constenla Umaña et al. (1998: 38). 


\section{Bibliografía}

Constenla Umaña, Adolfo. 1991. Las lenguas del Área Intermedia: introducción a su estudio areal. San José: Editorial de la Universidad de Costa Rica.

Constenla Umaña, Adolfo et al. 1998. Curso Básico de Bribri. San José: Editorial de la Universidad de Costa Rica.

Halliday, Michael. 1970. “Language structure and language function”. En: Webster (ed.). 2002, 173- 195.

1973. Explorations in the functions of language. Edward London: Arnold.

2004. An Introduction to Functional Grammar ( $3^{\text {rd }}$ ed. revised by C. Matthiessen). London: Hodder Arnold.

Halliday, Michael y Christian Matthiessen. 2006. Construing experience through meaning: a language-based approach to cognition. London: Continuum.

Jara Murillo, Carla Victoria. 1993. I ttè. Historias Bribris. San José: Editorial de la Universidad de Costa Rica.

1995. Text and context of the Sũwõ': Bribri Oral Tradition. PhD dissertation. Louisiana State University.

1998. "Estructura de la conversación y diálogo de géneros en la lengua bribri (chibcha)". Memoria del IV Encuentro de Lingüística en el Noroeste. México: Universidad de Sonora.

2002. "Tipología del orden de palabras en bribri” Revista de Filología y Lingüística. XXVIII (2). Universidad de Costa Rica.

2004a. "Observaciones para el estudio dialectológico de la lengua bribri”. Estudios de Lingüística Chibcha, XXIII. Universidad de Costa Rica.

2004b. "Hispanismos en la conversación bribri (familia chibcha)". Memoria del XIII Congreso internacional de la Asociación de Lingüística y Filología de América Latina (ALFAL). Universidad de Costa Rica.

Jara Murillo, Carla Victoria y Alí García Segura. 2008. Cargos tradicionales del pueblo bribri: Sĩõ’tãm̃ - Óköm - Awá. San José: Instituto Costarricense de Electricidad.

Martin, J.R, Christian Matthiessen, y Claire Painter. 1997. Working with Functional Grammar. London: Arnold.

Pacchiarotti, Sara. 2009. "La cláusula como intercambio en bribri según la gramática sistémico-funcional”. Tesis de Maestría en Lingüística: Universidad de Costa Rica.

Webster (ed.). 2002. On grammar. London: Continuum. 